\title{
Die Modularisierung der Berufsausbildung verbessert die Chancen von Jugendlichen auf dem Ausbildungsmarkt
}

\author{
DIETER EULER
}

\section{Trotz demografischer Entwicklung Gefahr der sozialen Exklusion}

Seit vielen Jahren gelingt es einer großen Zahl von Jugendlichen nicht oder nur mit zeitlicher Verzögerung, nach der Pflichtschulzeit eine Berufsausbildung aufzunehmen. Zwischen den allgemeinbildenden Schulen und der Berufsausbildung hat sich ein Übergangssektor etabliert, in dem im Jahr 2011 ca. 294.000 Jugendliche in einer Vielzahl von Maßnahmen untergebracht waren. Viele bleiben im Status des Ungelernten zurück - aktuell sind dies ca. 1,44 Mio. Personen in der Altersgruppe der 20- bis 29-Jährigen. Die Hoffnung, die demografische Entwicklung würde Betriebe zukünftig verstärkt animieren, Ausbildungsplätze auch mit Jugendlichen mit niedrigeren Schulabschlüssen zu besetzen, wird unter Experten nicht geteilt. So münden die Berechnungen im Nationalen Bildungsbericht 2010 in die Aussage, dass die Zahl der Jugendlichen im Übergangssektor aufgrund der demografischen Entwicklung bis 2025 zwar zurückgehen wird, jedoch ohne Veränderungen und effektive Interventionen immer noch auf einem Niveau von ca. 238.000 Jugendlichen bestehen bleibt. ${ }^{\circ}$

Vor diesem Hintergrund besteht die begründete Gefahr, dass sich die soziale Exklusion der Ausbildungslosen bzw. Ungelernten verstärkt. Da offensichtlich die bisher erprobten Maßnahmen erfolglos blieben, stellt sich die Frage nach neuen Ansätzen. Die Modularisierung stellt ein mögliches Mittel dar, der Gefahr einer sich verfestigenden Exklusion eines großen Teils der jugendlichen Schulabgänger entgegenzuwirken. Die Frage lautet: Wie können durch eine modulare Strukturierung der Berufsausbildung für diese gefährdeten Jugendlichen Wege geschaffen werden, die den Einstieg in eine Berufsausbildung erleichtern und sie ohne Zeitverlust zu einem anerkannten Berufsausbildungsabschluss führen? Mit dem Konzept der Ausbildungsbausteine wird im Folgenden eine konkrete Variante der
Modularisierung skizziert und durch die Diskussion aktueller Erprobungsbeispiele empirisch untermauert.

\section{Was (genau) meint Modularisierung?}

Das Konzept einer Modularisierung wird häufig missverstanden - angesichts der möglichen Zielbezüge und Ausprägungsformen ist dies auch nicht erstaunlich. Vor diesem Hintergrund erfordert eine rationale Diskussion zunächst eine Klärung der Bezugspunkte. Zum einen: Die Potenziale einer modularen Strukturierung von Ausbildungsgängen ließen sich auf unterschiedliche Zielkriterien hin diskutieren - hier steht die Ausprägung des Konzepts zur Erreichung einer besseren sozialen Integration gefährdeter Jugendlicher in die Berufsausbildung im Fokus. Zum anderen: Es geht nicht um Mikromodule von wenigen Tagen, wie sie teilweise im englischen und schottischen National Vocational Qualification (NVQ)-System zum Einsatz kommen. Vielmehr wird für die modulare Strukturierung von Ausbildungsgängen in der deutschen Berufsausbildung von einer übersichtlichen Zahl von fünf bis acht Ausbildungsbausteinen für einen Ausbildungsgang ausgegangen. Ausbildungsbausteine sind dabei abgegrenzte und bundesweit standardisierte Einheiten innerhalb der curricularen Gesamtstruktur eines Ausbildungsberufsbilds. Die einzelnen Bausteine entstehen aus einem ganzheitlichen Ausbildungsberufsbild, umgekehrt repräsentieren sie in ihrer Gesamtheit die Einheit des Berufsbilds. Erst die Absolvierung aller Bausteine begründet eine ganzheitliche, qualifizierte Berufsausbildung. Daraus ergibt sich, dass das Berufsprinzip als konstitutive Grundlage der dualen Berufsausbildung erhalten bleibt.

Durch die Einführung von Ausbildungsbausteinen in der dualen Ausbildung wird eine verbesserte horizontale und vertikale Verzahnung der Ausbildung mit vor- und nachgelagerten sowie parallelen vollzeitschulischen Bildungsange- boten ermöglicht. Dadurch verbessern sich u. a die Möglichkeiten, die Lernangebote auf die Voraussetzungen von Jugendlichen abzustimmen, die als vom Ausbildungsausschluss gefährdet gelten: Sie könnten in bewältigbaren Etappen zielgerichtet den Aufstieg zum Ausbildungsabschluss in Angriff nehmen.

\section{Welche Jugendlichen sind betroffen?}

Bei den gefährdeten Jugendlichen ließe sich hinsichtlich der vorhandenen Ausbildungsvoraussetzungen ein breites Kontinuum aufspannen, auf dem sich an dem einen Ende solche Jugendliche befinden, die sich vom Gros der Auszubildenden in einer dualen Ausbildung nicht unterscheiden und bei denen sich keine Hinweise auf besondere Schwierigkeiten zur erfolgreichen Absolvierung der Ausbildung finden. Am anderen Ende des Spektrums stehen Jugendliche mit einem spezifischen Förderbedarf etwa kognitiver oder sozialer Art, woraus sich jedoch nicht zwangsläufig eine Separierung oder Exklusion in dedizierte Vorbereitungsmaßnahmen ableiten lässt. Vielmehr ist zu berücksichtigen, dass mit der Separierung die Gefahr einer Stigmatisierung und negativen Selbstattribuierung wächst. Während die Schule mit ihren primär abstrakt-kognitiven Anforderungen für diese Jugendlichen häufig den rutschigen Abhang darstellt, auf dem sie keinen Halt mehr finden, sind sie außerhalb der Schule durchaus aktiv und engagiert. Dort suchen sie einen Raum der Bewährung, in dem der Zusammenhang zwischen dem eigenen Tun und persönlichem Erfolg sichtbar wird. Vor diesem Hintergrund sind schlechte Schulnoten zwar

Vgl. Autorengruppe Bildungsberichterstattung (2010): Bildung in Deutschland 2010. Ein indikatorengestützter Bericht mit einer Analyse zu Perspektiven des Bildungswesens im demografischen Wandel, Bielefeld. 
eine Hypothek für die mögliche Bewältigung von Herausforderungen in einer Ausbildung, doch könnten gerade die praktischen Dimensionen einer Ausbildung und Erfahrungen des Kompetenzerlebens jenseits kognitiver Abstraktionen den Jugendlichen neue Zugänge und Motivationsquellen auf ihrem Bildungsweg erschließen.

Die Ausführungen zeigen, dass die Verdichtung auf einzelne Merkmale wie der Schulabschluss, die Nationalität bzw. der Migrationshintergrund oder gar fragwürdige Etikettierungen wie „fehlende Ausbildungsreife“ den Voraussetzungen, insbesondere aber auch den Potenzialen und möglichen Stärken dieser Jugendlichen nicht gerecht werden können.

\section{Modularisierung mit besonderen Potenzialen}

Ausgangspunkt der Überlegungen ist die Frage: Wie müssen Ausbildungsformen gestaltet sein, um auch Jugendliche mit Förderbedarf ohne Zeitverlust zu einem Ausbildungsabschluss zu führen? Die Berufsausbildung für gefährdete Jugendliche wäre dann so zu gestalten, dass sie ihren kognitiven, motivationalen, sozialen u. a. Bedingungen gerecht wird. Gestaltungskomponenten wären dabei u. a. flexible Formen der Ausbildungsdauer, Ausbildungsbausteine und Teilzertifizierungen sowie individuelle Unterstützungsformen. Idealerweise verliefe dies im Rahmen einer (unterstützten) betrieblichen Berufsausbildung - fehlen hier jedoch entsprechende betriebliche Ausbildungsangebote, so wären subsidiär betriebsnahe Ausbildungsformen in schulischer, über- oder außerbetrieblicher Trägerschaft und Verantwortung zu schaffen. Ziel wäre eine Berufsausbildung für alle ausbildungswilligen Jugendlichen, möglichst bis zum Ausbildungsabschluss der Kammer, in jedem Fall aber bis zu einem individuell höchstmöglichen Teilabschluss.

Was leistet die Modularisierung in diesem Rahmen? Durch die Unterteilung in Module können die Lernergebnisse nicht erst am Ende der Ausbildung, sondern nach jedem Ausbildungsbaustein zertifiziert werden. Für die Jugendlichen wären damit die angestrebten Handlungskompetenzen transparent, sie hätten eine klare Zielorientierung. Jugendliche, die eine Ausbildung abbzw. unterbrechen müssen, hätten immerhin anerkannte Zwischenabschlüsse erreicht, an die sie später anknüpfen und die sie dann auch weiter ausbauen können. Sie verfügen in einem solchen Fall über einen anerkannten und durch Betriebe nachvollziehbaren Nachweis einer beruflichen Teilqualifikation, der sie auf dem
Arbeitsmarkt immerhin aus dem Status eines Ungelernten herauslösen würde und der den Einstieg in das Beschäftigungssystem erleichtern könnte. Durch die Lernerfolgsprüfung am Ende eines Ausbildungsbausteins könnten zum einen Lücken bei den Jugendlichen gezielter diagnostiziert und durch passgenaue pädagogische Fördermaßnahmen geschlossen werden. Zum anderen bietet die Prüfung und Zertifizierung von Handlungskompetenzen Anlass zu häufigeren Erfolgserlebnissen mit den daraus resultierenden Motivierungspotenzialen. Durch die Ausrichtung von Maßnahmen auf einen Ausbildungsabschluss verlören diese weitgehend ihre Stigmatisierungswirkung. Sie würden nicht mehr als Warteschleifen für „schwierige Jugendliche“ wahrgenommen, sondern formal und faktisch als vollwertige Ausbildungsformen.

\section{Die Potenziale lassen sich umsetzen}

Die skizzierten Überlegungen bilden den Kern einer Studie, ${ }^{0}$ die vor einigen Jahren im Innovationskreis Berufliche Bildung vorgestellt wurde. Mittlerweile liegen praktische Umsetzungserprobungen vor, die eine erste Einschätzung der dargestellten Potenziale erlauben.

Die Erprobung von Ausbildungsbausteinen wurde von der Bundesregierung 2009 als Ziel in den Koalitionsvertrag aufgenommen. Die Umsetzung dieser Zielsetzung soll in dem seit 2009 geförderten Programm „Jobstarter Connect“ erfolgen. In zunächst 14 Ausbildungsberufen werden außerhalb einer betrieblichen Ausbildung bundesweit einheitliche Ausbildungsbausteine erprobt. Das Programm sieht vor, die über die Absolvierung der Ausbildungsbausteine erworbenen beruflichen Handlungskompetenzen transparent zu dokumentieren und damit für die Jugendlichen besser verwertbar zu machen. Die Bescheinigung der Kompetenzen erfolgt zwar nicht auf der Grundlage einheitlicher Prüfungs- und Zertifizierungsstandards, dennoch soll die vom Träger ausgestellte Bescheinigung dazu beitragen, dass die Übernahme des Jugendlichen in eine betriebliche Ausbildung und die Anrechnung seiner erbrachten Ausbildungsleistungen erleichtert werden. Bis Ende 2011 nahmen in 40 regionalen Projekten rund 3.000 Jugendliche an einer Qualifizierung über Ausbildungsbausteine teil. Als Ergebnisse der durchgeführten Evaluation lassen sich u. a. die folgenden Erkenntnisse festhalten:

- eine Qualitätsverbesserung und stärkere duale Orientierung der Maßnahmen des Übergangsbereichs;
- gezielte Abstimmungsprozesse zwischen den Lernorten zur Sicherung des Kompetenzerwerbs; - eine verbesserte Lernergebnisorientierung in den Ausbildungsmaßnahmen, verbunden mit einer Verbesserung der Rückkopplung von Lernerfolgen für die Jugendlichen (neue Feedbackkultur);

- Steigerung der Motivation und des Selbstwertgefühls der jugendlichen Teilnehmer.

Modulare Ausbildungsformen auf der Grundlage von Ausbildungsbausteinen wurden zudem auf der Ebene von Bundesländern erprobt und evaluiert. Ein Beispiel stellt das in Nordrhein-Westfalen durchgeführte Projekt „3. Weg in die Berufsausbildung " dar. ${ }^{3}$ Ziel ist die berufliche Qualifizierung bis hin zum Berufsabschluss über zertifizierbare Ausbildungsbausteine. Die Grundlage bildet ein regulärer Ausbildungsvertrag mit dem Bildungsträger nach Berufsbildungsgesetz bzw. Handwerksordnung. Ein Drittel bis die Hälfte der Ausbildungszeit wird im Betrieb absolviert. Der „3. Weg“ lässt Unterbrechungen der Ausbildung von bis zu sechs Monaten zu. Insgesamt haben Auszubildende die Option auf eine Verlängerung der Ausbildung auf bis zu fünf Jahren. Die Ausbildungsbausteine sollen als Instrument zur Untergliederung der Ausbildung eine individualisierte und flexibilisierte Ausbildung ermöglichen. Für jeden Ausbildungsbaustein wird durch den Ausbildenden eine Leistungsbewertung vorgenommen. Über ein Bildungscoaching sollen die Jugendlichen eine ausbildungsbegleitende Stabilisierung erhalten. Die wissenschaftliche Begleitung fasste die Ergebnisse aus der Evaluation des ersten Pilotjahrgangs wie folgt zusammen: „Unter Berücksichtigung dessen, dass den Jugendlichen und jungen Erwachsenen eine mangelnde Ausbildungsreife im Vorfeld attestiert wurde, ist es als durchschlagender Erfolg des Pilotprojektes zu werten, dass etwa $40 \%$ der Auszubildenden des ersten Durchgangs die Ausbildung planmäßig beendet haben und mit einem qualifizierten Berufsabschluss in den Arbeitsmarkt eintreten können. Für die Zielerreichung des „3. Weg“ spricht auch, dass 88 Prozent der Jugendlichen und jungen Erwachsenen, die sich

(2) Vgl. Euler, D./Severing, E. (2007): Flexible Ausbildungswege in der Berufsbildung, Bielefeld.

(3) Vgl. Gesellschaft für Innovationsforschung und Beratung (GIP) (2010): Evaluation des Pilotprojekts "3. Weg in der Berufsausbildung in NRW", Abschlussbericht, Internes Manuskript, Berlin. 
zur Abschlussprüfung anmelden, die Berufsausbildung erfolgreich abschließen."(*)

Die bislang umfassendste Umsetzung einer modular strukturierten Berufsausbildung findet derzeit in Luxemburg statt. Im Rahmen einer umfassenden Reform der Berufsbildung wird die Berufsausbildung u. a. auf eine modulare Struktur umgestellt. Dabei werden sämtliche Ausbildungsgänge in Bausteinen strukturiert, die in einer zeitlich begrenzten Phase von sechs bis zwölf Monaten absolviert werden und zu abgeschlossenen und geprüften „Teilqualifikationen“ führen. Damit besitzt ein Baustein eine gewisse Komplexität, in der Regel repräsentiert er einen größeren Arbeitsbereich innerhalb des Berufs. Ein Baustein wird zumeist weiter in Module aufgeteilt, die jeweils eine sinnvolle Ausbildungseinheit darstellen. Jedes Modul wird über Kompetenzen beschrieben, die von den Modulverantwortlichen im jeweiligen Lernort zu vermitteln und eigenständig zu prüfen sind. Je nach Ausbildungsberuf und -niveau finden die Ausbildung sowie die darauf bezogenen Prüfungen mit unterschiedlichen Anteilen in Schule und Betrieb statt. Die Zuordnung der Modul- und Prüfungsverantwortung zwischen Schule und Betrieb wird im Rahmen der Entwicklung der Ordnungsgrundlagen entschieden. Neben den modulbezogenen Prüfungen werden zur Mitte sowie zum Ende der Ausbildung zwei Integrationsprojekte vorgesehen, in denen die Bearbeitung von bausteinübergreifenden, berufsbezogenen Arbeitsaufgaben im Mittelpunkt steht. Die Integrationsprojekte werden zentral von Prüfungsausschüssen durchgeführt, die durch das Ministerium eingesetzt werden. Diese integrativen Prüfungen heben auf die Feststellung von modulübergreifenden beruflichen Handlungskompetenzen ab. Integrationsprojekte müssen erfolgreich absolviert werden, um die Berufsausbildung insgesamt zu bestehen. Die skizzierte Strukturierung der Ordnungsgrundlagen in Form von Bausteinen und Modulen führt zu Ausbildungseinheiten mit einer gewissen Komplexität, wodurch der Gefahr einer Fragmentarisierung des Ausbildungsgangs und damit einer Gefährdung der Einheitlichkeit des Berufsbilds entgegengewirkt werden kann. Wenn nach Abschluss einzelner Bausteine durch geeignete Prüfungen die Kompetenzen festgestellt wurden, dann ergeben sich daraus drei wesentliche Konsequenzen:

(1) Bei Nicht-Bestehen einzelner Module muss nicht wie bisher ein ganzes Ausbildungsjahr wiederholt werden, sondern in den problematischen Modulen können gezielt spezifische Lücken aufgearbeitet werden.

(2) Bei Ausbildungsabbruch besitzt der Auszubildende bereits einen Nachweis über die Bereiche der Ausbildung, in denen er die vorgesehenen Kompetenzen im Rahmen der bestandenen Module erworben hat. Dies erleichtert die Möglichkeit der Wiederaufnahme der Berufsausbildung und unterstützt eine gezielte Nachqualifizierung.

(3) Mögliche Ausbildungsschwierigkeiten werden bereits am Ende eines Bausteins deutlich. Dadurch ist es früher und gezielter möglich, auf Leistungsschwächere einzugehen und Ausbildungsschwierigkeiten mit gezielten Hilfen zu begegnen.

\section{Einwände und Ausblicke}

Gelegentlich wird das Argument vorgetragen, dass die Einführung von Ausbildungsbausteinen schleichend zur Auflösung des Berufsprinzips führe. Unabhängig von dem fehlenden Beleg, dass berufliche Handlungskompetenz nur innerhalb eines dualen Ausbildungsgangs erreichbar

Vgl. GIP (2010), a.a.O., S. 225. ist bzw. durch die aktuelle Ausprägung dualer Ausbildungsverhältnisse in der Regel auch tatsächlich entsteht, gibt es weder theoretisch noch praktisch eine stichhaltige Begründung, weshalb eine andersartige Strukturierung von Ausbildung und Prüfung die Erreichung der mit dem Berufsprinzip verbundenen Merkmale verfehlen sollte.

Die vorgestellten Überlegungen richten sich nicht gegen die duale Berufsausbildung. Dort, wo diese in quantitativer und qualitativer Hin sicht funktioniert, gibt es wenig Grund für neue Strukturmodelle. Wenn jedoch, wie nunmehr seit nahezu zwei Dekaden, eine große Gruppe von Jugendlichen an den Katzentisch des Bildungssystems verbannt wird oder gar dauerhaft von einer sozialen Exklusion bedroht ist, dann erscheinen neue Konzepte nicht nur möglich, sondern gesellschaftspolitisch geboten. Wenn man andere als die gewohnten Antworten haben will, darf man nicht auf die gewohnte Art fragen. Eine Weiterentwicklung der Berufsbildung lässt sich dabei nicht getrennt auf das Übergangssystem auf der einen Seite oder auf das duale System auf der anderen Seite fragmentieren. Viele der Jugendlichen bewegen sich in beiden Bereichen und büßen für die Versäumnisse der berufsbildungspolitisch Verantwortlichen. Es wird Zeit, zusammenzudenken, was zusammengehört oder wie heißt es so treffend: Wer nicht mit der Zeit geht, der geht mit der Zeit.

\section{AUTOR}

DIETER EULER, Prof. Dr., Lehrstuhl für Wirtschaftspädagogik und Bildungsmanagement an der Universität St. Gallen. Arbeitsschwerpunkte: Förderung und Evaluation von Sozialkompetenzen, Modernisierung der Berufsbildung, der Hochschulentwicklung sowie des Bildungsmanagements.

dieter.euler@unisg.ch 